\title{
Improving the Shelf-Life of Fruits Using Chitosan and Chitosan-Based Glycodynamer Coatings ${ }^{+}$
}

\author{
Ioana-Alexandra Bala ${ }^{1}$, Cătălina-Diana Cristea ${ }^{1}$, Luminița Dimitriu ${ }^{1}$, Diana \\ Constantinescu-Aruxandei 1,* , Luminița Marin ${ }^{2}$, Sanda-Maria Doncea ${ }^{1}$ and Florin Oancea ${ }^{1, *}$ \\ 1 INDCP-ICECHIM Bucharest, 202 Spl. Independentei, 6th district, 060021 Bucharest, Romania; \\ balaioana97@yahoo.com (I.-A.B.); cristeacatalina95@gmail.com (C.-D.C.); lmarin@icmpp.ro (L.D.); \\ sandamariadocea@gmail.com; (S.-M.D.) \\ 2 Petru Poni, Institute of Macromolecular Chemistry of Romanian Academy, 700487 Iasi, Romania; \\ lmarin@icmpp.ro \\ * Correspondence: diana.constantinescu@icechim-rezultate.ro (D.C.-A.); florin.oancea@icechim.ro (F.O.) \\ + Presented at the15th International Symposium "Priorities of Chemistry for a Sustainable Development" \\ PRIOCHEM, Bucharest, Romania, 30th October-1st November 2019.
}

Published: 17 October 2019

Keywords: chitosan; glycodynameric hydrogel; edible bioactive coating

The study aims to develop an edible bioactive coating in the form of a chitosan glycodynameric film, which is intended to protect fresh fruits (berry fruits, apples), during storage. A solution of $2 \%$ chitosan in acetic acid $(0.7 \%)$, was cross-linked with $1 \%$ citral prepared in ethanol, generating a glycodynameric structure [1]. The citral solution was added to chitosan under continuous magnetic stirring at $55^{\circ} \mathrm{C}$. The glycodynameric feature (reversible/dynamic covalent bonds) is determined by the competition between the imine formation (amino groups of chitosan-aldehyde group of citral) with citral aliphatic side chains' self-organization into supramolecular layered architectures [2]. The resulted glycodynameric structures combine excellent mechanical properties with stimuli-controlled transitions [3]. Two types of film coating were applied on berry fruits, chitosan coating and glycodynamer coating. The fruits with and without coating, the chitosan and the glycodynameric films were characterized by FTIR spectroscopy. The decay of strawberries was reduced significantly when berries were either coated with chitosan (reference control) or with glycodynameric coating. The early signs of mold development on strawberries appeared after 9 days of storage at room temperature. Both the chitosan and the glycodynameric coating reduced the fungal decay, the glycodynamer being more efficient, probably due to the presence of citral. The FTIR spectral bands characteristic to chitosan and glycodynamer were observed on the surface of fruits, but the glycodynamer stability, in time, needs to be optimized. In conclusion, our study indicates that preservative coating based on glycodynamers has a potential to prolong storage life and control the fungal decay of fruits. Further studies with other fruits and different glycodynamers are needed.

Acknowledgments: The work was supported by Ministry of Research and Innovation, Project 10PCCDI/2018 Closing the loop into bioeconomy value-chains by manufacturing innovative bioproducts-PRO-SPER, funded by UEFISCDI and by Project PFE 31/2018, Enhancing the INCDCP-ICECHIM research and innovation potential within the field of inter-disciplinary and cross-sectoral key enabling technologies-TRANS-CHEM, Project PN.19.23.01.03. 


\section{References}

1. Perdones, A.; Sánchez-González, L.; Chiralt, A.; Vargas, M. Effect of chitosan-lemon essential oil coatings on storage-keeping quality of strawberry. Postharvest Biol. Technol. 2012, 32-41, doi:10.1016/j.postharvbio.2012.04.002.

2. Picchioni, F.; Muljana, H. Hydrogels Based on Dynamic Covalent and Non Covalent Bonds: A Chemistry Perspective. Gels 2018, 4, 21. doi:10.3390/gels4010021.

3. Marin, L.; Ailincai, D.; Morariu, S.; Tartau-Mititelu, L. Development of biocompatible glycodynameric hydrogels joining two natural motifs by dynamic constitutional chemistry. Carbohydr. Polym. 2017, 170, 6071, doi:10.1016/j.carbpol.2017.04.055.

(C) 2019 by the authors. Licensee MDPI, Basel, Switzerland. This article is an open access article distributed under the terms and conditions of the Creative Commons Attribution (CC BY) license (http://creativecommons.org/licenses/by/4.0/). 